Silval Fernando Cardoso Zabaglia 1 Adriana Orcesi Pedro 1

Aarão Mendes Pinto Neto 1

Telma Guarisi 1

Lucia Helena Simões da Costa Paiva 1

Eduardo Lane 1

\section{Estudo exploratório da associação entre o perfil lipídico e a densidade mineral óssea em mulheres menopausadas, em hospital de referência de Campinas}

\author{
Association between lipid profile and bone \\ mineral density in post-menopausal women
}

1 Departamento de Tocoginecologia, Faculdade de Ciências Médicas, Centro de Atenção Integral à Saúde da Mulher, Universidade Estadual de Campinas. Rua Alexander Fleming 101, Cidade Universitária Zeferino Vaz. C. P. 6081, Campinas, SP 13083-970, Brasil.
Abstract A total of 72 postmenopausal patients presenting no risk factors for cardiovascular disease nor osteoporosis, were studied. The study evaluated total serum cholesterol and fractions and bone mass by densitometry of the lumbar spine and femur using a Lunar-DPX. There was no association between lipid profile variables and bone mineral density, except for high density lipoprotein (HDL), which showed an inverse correlation $(p=0.001)$. Multiple regression showed that total cholesterol levels higher than $240 \mathrm{mg} \%$ had a positive association with BMD ( $\mathrm{p}=$ 0.026). In addition, the ratio between LDL and HDL (Castelli 2 index) showed a negative association with BMD ( $p=0.002)$. The diagnostic validation test showed that all lipid profilevariables had low sensitivity and specificity as indi cators for osteoporosis. The conclusions were that lipid profilevariables did not show a significant association with bone mass and could not be used as indicators for bone mineral density.

Key words Menopause; Osteoporosis; Cardiovascular Disease

Resumo O objetivo deste estudo foi avaliar a possível associação entre al gumas variáveis do perfil li pídico ea densi dade mineral óssea e se estas variáveis poderiam ser usadas como indicadoras de massa óssea em mulheres menopausadas, atendi das no ambulatório de menopausa do CAISM-Unicamp, no ano de 1995. Estudaram-se 72 pacientes pós-menopausadas, sem fatores de risco para doenças cardi ovasculares e para osteoporose, por meio da dosagem de colesterol total e frações e da avaliação da densidade mineral óssea por densitometria óssea em aparelho Lunar DPX (Dexa). Dentre as variávei s do perfil li pídico, a densi dade mineral óssea associou-se inversamente à li poproteína HDL de alta densi dade $(p=0,001)$. A análise de regressão múlti pla observou que níveis de colesterol total acima de $240 \mathrm{mg} \%$ associaram-se a menor densidade mineral óssea $(p=0,026)$. A razão entre a lipoproteína de baixa densi dade e a lipoproteína de al ta densi dade (índi ce de Castel li 2) correl aci onou-se posi tivamente com a densi dade mineral óssea ( $p=0,002)$. O teste de vali dação diagnósti ca mostrou que todas as variáveis do perfil li pídico apresentaram baixa sensi bilidade e especifi ci dade como indicadoras de di minui ção de massa óssea. Concl ui-se que, a pesar de al gumas variáveis do perfil li pídi co apresentarem associação estatisticamente si gnifi cativa com a massa óssea, el as foram contradi tórias e não têm boa capacidade diagnóstica.

Palavras-chave Menopausa; Osteoporose; Sistema Cardiovascular 


\section{Introdução}

Com o aumento significativo da expectativa de vida das mulheres e, conseqüentemente, das doenças crônicas, como as doenças cardiovasculares e a osteoporose, muitos profissionais de saúde têm solicitado a realização do perfil lipídico e da avaliação da massa óssea, quase que rotineiramente, para atendimento de pacientes climatéricas.

Entretanto, estes exames complementares, em geral, são financeiramente onerosos para a mulher e mesmo para os serviços públicos de saúde, visto que são realizados, de uma maneira geral, anualmente em todas as mulheres em acompanhamento pelo climatério. Em Campinas, a estimativa de custo, em dólares, para a realização do perfil lipídico plasmático completo está entre US $\$ 35$ e US $\$ 50$, e entre US $\$ 75$ e US $\$ 100$ para a densintometria óssea. Nestes valores não estão computados os gastos que as mulheres têm com transporte e alimentação para cada dia que comparecem ao hospital a fim de realizar os exames.

Bagbozan et al. (1993) e Soo et al. (1993) estudaram, em mulheres menopausadas, a relação entre o colesterol total e o LDL colesterol com a densidade mineral óssea medida na coluna lombar. Esses autores sugeriram que muIheres com colesterol total e LDL colesterol elevados, ou seja, com risco para doenças cardiovasculares, também apresentaram massa óssea diminuída em coluna lombar. Não foram encontrados outros artigos referentes a esta associação na literatura, demonstrando ser um assunto novo e necessitando de novas investigações clínicas.

Tais resultados, juntamente com a idéia de que talvez o perfil lipídico pudesse substituir a densitometria óssea no rastreamento da osteoporose entre mulheres menopausadas, motivaram a realização deste estudo, que visa à simplificação da propedêutica necessária ao atendimento da mulher climatérica, provendo uma diminuição nos gastos, tanto para o sistema de saúde, como para a própria paciente, não descuidando da segurança e da qualidade do atendimento.

\section{Casuística e métodos}

Foram estudadas 72 mulheres menopausadas, com idade entre 41 e 65 anos, admitidas no ambulatório de menopausa do CAISM/Unicamp (Centro de Atenção Integral à Saúde da Mulher/Universidade Estadual de Campinas), no ano de 1995, ano em que foram admitidas quatrocentas mulheres no serviço. Todas as pacientes encontravam-se em amenorréia por um período de tempo superior a um ano e sem uso naquele período ou pregresso de terapia de reposição hormonal. Não participaram do estudo pacientes histerectomizadas ou portadoras de al guma patologia crônica, como hipertensão arterial, diabetes mellitus, artropatia, tireoidopatia (hiper ou hipotireoidismo), cardiopatia (arritmias e isquêmicas) etc., ou de neoplasia maligna. Foram utilizados estes critérios de exclusão para que a pesquisa de algumas destas patologias, bem como o uso da terapia de reposição hormonal, não influenciassem no resultado tanto do perfil lipídico, quanto da densitometria óssea. Não houve nenhuma recusa e finalizamos a sel eção quando o tamanho amostral necessário foi atingido.

O tamanho da amostra foi calculado em 61 pacientes, considerando-se $1,16 \mathrm{~g} / \mathrm{cm}^{2}$ (Kish, 1965) a média da densidade mineral óssea na coluna lombar de mulheres pós-menopausadas na população, com desvio-padrão (D. P.) de $0,16 \mathrm{~g} / \mathrm{cm}^{2}$, erro alfa de 0,05 , e considerando-se 0,04 (Souza \& Schneider, 1993) a diferença desejada entre a média de densidade mineral óssea em mulheres pós-menopausadas na amostra estudada e na população geral.

Para a realização do perfil lipídico colheram-se $10 \mathrm{ml}$ de sangue, com agul ha e seringa descartáveis, após jejum total de 14 horas, na sala de coleta do Hospital das Clínicas - Unicamp. Posteriormente, o sangue foi analisado por técnicos e conferido pelo patologista clínico responsável, sendo, então, emitido laudo em duplicata, conforme técnica habitual, pelo Laboratório de Patologia Clínica do HC-Unicamp.

A massa óssea foi medida através da densitometria óssea, exame realizado com aparelho Lunar DPX, por especialistas do setor de Medicina Nuclear do HC-Unicamp, com emissão de laudo computadorizado. Escolheu-se para o estudo a medida de densidade mineral óssea em três locais do fêmur (colo do fêmur, triângulo de Wards e trocânter) e coluna lombar (utilizando o espaço entre $L_{2}-L_{4}$ ).

A análise dos dados foi realizada em duas etapas. Na primeira, testou-se a diferença entre as médias da densidade mineral óssea nas diversas localizações segundo valores estratificados do perfil lipídico, utilizando-se o teste t de Student e a Anova. Posteriormente, realizamos a análise de regressão múltipla, utilizando como variável dependente a densidade mineral óssea na coluna lombar $\left(\mathrm{L}_{2}-\mathrm{L}_{4}\right)$, colo do fêmur, triângulo de Wards e trocânter, referentes aos quatros modelos desenvolvidos, e como variável independente, o perfil lipídico, repre- 
sentado pelo colesterol total e o índice de Castelli 2 (Cox, 1970).

Na segunda etapa, realizamos uma validação do perfil lipídico como teste diagnóstico para osteoporose, considerando a densitometria óssea como padrão ouro. Calculamos sensibilidade, especificidade, traçamos a curva ROC (Recevier Operator Characteristic Curve) e determinamos o ponto de corte para cada uma das variáveis do perfil lipídico analisadas. Testes de bom poder discriminatório concentramse no canto superior esquerdo da curva ROC, e testes de menor poder discriminatório têm curvas mais próximas à diagonal (Fletcher et al., 1991).

\section{Resultados}

\section{Caracterização da população}

A idade das 72 pacientes estudadas variou entre 41 e 65 anos, com média de 52 anos (DP \pm $4,74)$. A média etária para a ocorrência da menopausa foi de 48 anos (DP $\pm 3,45$ ), sendo que, para mais da metade das pacientes avaliadas, a menopausa ocorreu entre 51 e 55 anos. $O$ tempo médio após a menopausa foi de quatro anos, observando-se maior percentual de muIheres menopausadas há menos de dois anos (Tabelas 1, 2, 3).

Os valores médios dos indicadores do perfil lipídico apresentaram-se dentro da faixa da normalidade segundo valores de referência, com exceção do colesterol total e do HDL colesterol. O colesterol total apresentou média de $213 \mathrm{mg} \%$, o que pode ser considerado acima dos valores padronizados como normais. $O$ HDL colesterol apresentou valores médios de $59,67 \mathrm{mg} \%$, considerado abaixo do limite da normalidade.

Em relação aos valores médios da densidade mineral óssea nas diferentes regiões do fêmur e da coluna lombar, observou-se que o local de maior densidade foi a coluna lombar, seguida pelo colo do fêmur, triângulo deWards e trocânter.

A distribuição percentual da densidade mineral óssea segundo o diagnóstico de normal, oesteopenia e osteoporose mostrou que a maioria das pacientes encontrava-se na faixa de normalidade no fêmur, triângulo de Wards e trocânter, e mais da metade delas apresentaram alterações na coluna lombar (Tabela 4).
Tabela 1

Valores médios da idade, idade na menopausa e tempo de menopausa, em anos $(n=72)$.

\begin{tabular}{lcccc}
\hline Variáveis & Mínimo & Máximo & X & D. P. \\
\hline Idade & 41 & 65 & 52,14 & 4,74 \\
Idade na menopausa & 38 & 56 & 48,12 & 3,45 \\
Tempo de menopausa & 01 & 17 & 4,01 & 3,22 \\
\hline
\end{tabular}

Tabela 2

Distribuição percentual das mulheres segundo faixa etária.

\begin{tabular}{lrr}
\hline Idade (anos) & $\mathrm{n}$ & $\%$ \\
\hline 41 a 45 & 3 & 4,2 \\
46 a 50 & 25 & 34,7 \\
51 a 55 & 30 & 41,7 \\
56 a 60 & 8 & 11,2 \\
61 a 65 & 6 & 8,4 \\
Total & 72 & 100,0 \\
\hline
\end{tabular}

Tabela 3

Distribuição percentual das mulheres segundo o tempo de menopausa.

\begin{tabular}{lcc}
\hline Tempo (anos) & $\mathrm{n}$ & $\%$ \\
\hline Até 2 & 33 & 45,8 \\
2 a 5 & 24 & 33,4 \\
$\geq 6$ & 15 & 20,8 \\
Total & 72 & 100,0 \\
\hline
\end{tabular}

Tabela 4

Distribuição percentual da densidade mineral óssea segundo diagnóstico de normal, osteopenia e osteoporose $(n=73)$.

\begin{tabular}{|c|c|c|c|c|c|c|}
\hline \multirow[t]{2}{*}{ Localização } & \multicolumn{2}{|c|}{ Normal } & \multicolumn{2}{|c|}{ Osteopenia } & \multicolumn{2}{|c|}{ Osteoporose } \\
\hline & $\mathrm{n}$ & $\%$ & $\mathrm{n}$ & $\%$ & $n$ & $\%$ \\
\hline Colo do fêmur & 50 & 68,5 & 22 & 30 & 1 & 1,4 \\
\hline Triângulo de Wards & 41 & 56,1 & 29 & 39,7 & 3 & 4,1 \\
\hline Trocânter & 56 & 76,7 & 17 & 23,3 & 0 & 0 \\
\hline Coluna lombar & 35 & 47,9 & 28 & 38,5 & 10 & 13,7 \\
\hline
\end{tabular}


Associação entre o perfil lipídico e a densidade óssea

Quando realizamos a associação do perfil lipídico com a densidade mineral óssea, observamos que os menores valores médios da densidade mineral óssea corresponderam ao grupo de paciente com níveis plasmáticos médios de colesterol total maiores ou iguais a $240 \mathrm{mg} \%$. Essas diferenças não foram estatisticamente significativas (Tabela 5).

Para o HDL colesterol, a menor densidade mineral óssea foi observada para o grupo de pacientes com níveis médios de HDL colesterol iguais ou maiores de $80 \mathrm{mg} \%$, com diferença estatisticamente significativa em todos os locais (Tabela 6).

A média da densidade mineral óssea no fêmur e coluna lombar foi maior no grupo de pacientes com LDL colesterol entre 130-159mg\%, e menor no grupo com LDL colesterol igual ou maior de 159mg\%. Estas diferenças não foram estatisticamente significativas (Tabela 7).

$\mathrm{Na}$ associação entre a densidade mineral óssea em diferentes localizações e a relação co- lesterol total/HDL colesterol (Índice de Castelli 1), observamos maior média da densidade óssea no grupo de pacientes com Índice de Castelli 1 mai or ou igual a 4,4; entretanto, tais diferenças não foram significativas (Tabela 8).

Constatamos maiores médias da densidade mineral óssea nas diferentes localizações do fêmur e da coluna lombar em mulheres com Índice de Castelli 2 (relação LDL colesterol/ HDL colesterol) maior que 3,2; contudo, essas diferenças não foram significativas (Tabela 9).

A regressão linear múltipla mostrou associação do colesterol total com valores acima de $240 \mathrm{mg} \%$ e do Índice de Castelli 2 com a densidade mineral óssea no colo do fêmur, triângulo de Wards e trocânter. Os valores do colesterol total acima de $240 \mathrm{mg} \%$ associaram-se a menor densidade mineral óssea nestas localizações, correspondendo a uma associação inversa. Os níveis do Índice de Castelli 2 apresentaram uma associação direta, ou seja, quanto maiores os seus valores médios, maior a média da densidade mineral óssea. O modelo utilizando os valores médios da densidade mineral óssea na coluna lombar não apresentou associação es-

Tabela 5

Média e D. P. da densidade mineral óssea em diferentes localizações segundo os valores estratificados do colesterol total.

\begin{tabular}{|c|c|c|c|c|c|c|c|}
\hline \multirow[t]{3}{*}{ Localização } & \multicolumn{6}{|c|}{ Colesterol total (mg\%) } & \multirow[t]{3}{*}{$p^{*}$} \\
\hline & \multicolumn{2}{|c|}{ Até $200(n=28)$} & \multicolumn{2}{|c|}{200 a $239(n=28)$} & \multicolumn{2}{|c|}{$\geq 240(n=16)$} & \\
\hline & $x$ & D. P. & $x$ & D. P. & $x$ & D. P. & \\
\hline Colo do fêmur & 0,944 & 0,135 & 0,961 & 0,150 & 0,879 & 0,129 & 0,169 \\
\hline Triângulo de Wards & 0,808 & 0,145 & 0,837 & 0,162 & 0,734 & 0,166 & 0,089 \\
\hline Trocânter & 0,769 & 0,141 & 0,802 & 0,149 & 0,732 & 0,111 & 0,154 \\
\hline Coluna lombar & 1,083 & 0,159 & 1,048 & 0,307 & 1,049 & 0,179 & 0,735 \\
\hline
\end{tabular}

* Anova

Tabela 6

Média e D. P. da densidade mineral óssea em diferentes localizações segundo os valores estratificados do HDL colesterol.

\begin{tabular}{|c|c|c|c|c|c|}
\hline \multirow[t]{3}{*}{ Localização } & \multicolumn{4}{|c|}{ HDL colesterol (mg\%) } & \multirow[t]{3}{*}{$\mathrm{p}^{*}$} \\
\hline & \multicolumn{2}{|c|}{$<80(n=66)$} & \multicolumn{2}{|c|}{$\geq 80(n=6)$} & \\
\hline & $x$ & D. P. & $x$ & D. P. & \\
\hline Colo de fêmur & 0,951 & 0,134 & 0,764 & 0,095 & 0,001 \\
\hline Triângulo de Wards & 0,819 & 0,153 & 0,614 & 0,104 & 0,002 \\
\hline Trocânter & 0,789 & 0,133 & 0,596 & 0,067 & 0,001 \\
\hline Coluna lombar & 1,079 & 0,228 & 0,874 & 0,157 & 0,006 \\
\hline
\end{tabular}

* Teste t de Student 
Tabela 7

Média e D. P. da densidade mineral óssea em diferentes localizações segundo os valores estratificados do LDL colesterol.

\begin{tabular}{|c|c|c|c|c|c|c|c|}
\hline \multirow[t]{3}{*}{ Localização } & \multicolumn{6}{|c|}{ LDL colesterol (mg\%) } & \multirow[t]{3}{*}{$p^{*}$} \\
\hline & \multicolumn{2}{|c|}{$\leq 130(n=46)$} & \multicolumn{2}{|c|}{130 a $159(n=18)$} & \multicolumn{2}{|c|}{$\geq 159(n=8)$} & \\
\hline & X & D. P. & $x$ & D. P. & $x$ & D. P. & \\
\hline Colo do fêmur & 0,933 & 0,133 & 0,968 & 0,148 & 0,876 & 0,165 & 0,309 \\
\hline Triângulo de Wards & 0,794 & 0,142 & 0,858 & 0,168 & 0,729 & 0,211 & 0,129 \\
\hline Trocânter & 0,762 & 0,136 & 0,812 & 0,151 & 0,750 & 0,133 & 0,597 \\
\hline Coluna lombar & 1,044 & 0,240 & 1,129 & 0,195 & 1,017 & 0,299 & 0,332 \\
\hline
\end{tabular}

* Anova

tatisticamente significativa com os níveis plasmáticos do colesterol total acima de $240 \mathrm{mg} \%$ e com o Índice de Castelli 2 (Tabela 10).

Quando analisamos os valores estratificados do colesterol total como indicadores da diminuição da densidade mineral óssea no colo do fêmur e na coluna lombar, utilizando como padrão ouro a densitometria óssea, observamos que o melhor ponto de corte para as duas localizações foi 210mg\% (Figura 1). Mesmo para o melhor ponto de corte, a sensibilidade e especificidade foram baixas, tanto para o colo de fêmur, como para a coluna lombar.

Os valores estratificados do HDL colesterol como indicadores de diminuição da densidade mineral óssea no colo do fêmur e na coluna lombar também apresentaram sensibilidade e especificidade baixas, mesmo para o melhor ponto de corte, que foi de $50 \mathrm{mg} \%$ para as duas localizações (Figura 2).

Também os valores estratificados do LDL colesterol como indicadores de diminuição da densidade mineral óssea, tanto no colo do fêmur, quanto na coluna lombar, apresentaram sensibilidade e especificidade baixas para o melhor ponto de corte $(110 \mathrm{mg} \%)$ e para as duas localizações (Figura 3).

\section{Discussão}

A proposição principal deste estudo foi a de avaliar se existe associação entre al gumas variáveis do perfil lipídico e a densidade mineral óssea em mulheres menopausadas. Representa uma tentativa de verificar se mulheres com maior risco de desenvolver doenças cardiovasculares têm, também, um maior risco para osteoporose. Concluiu-se não ter sido o perfil lipídico um bom indicador da densidade óssea, pois observaram-se resultados contraditórios. Os resultados demonstraram que níveis plas-
Tabela 8

Média e D. P. da densidade mineral óssea em diferentes localizações segundo a relação colesterol total/HDL colesterol (índice de Castelli 1).

\begin{tabular}{|c|c|c|c|c|c|}
\hline \multirow[t]{3}{*}{ Localização } & \multicolumn{4}{|c|}{ Castelli $1(n=71)$} & \multirow[t]{3}{*}{$p^{*}$} \\
\hline & \multicolumn{2}{|c|}{$<4,4(n=57)$} & \multicolumn{2}{|c|}{$\geq 4,4(n=14)$} & \\
\hline & $x$ & D.P. & $x$ & D.P. & \\
\hline Colo de fêmur & 0,917 & 0,132 & 1,012 & 0,158 & 0,397 \\
\hline Triângulo de Wards & 0,783 & 0,137 & 0,885 & 0,217 & 0,135 \\
\hline Trocânter & 0,751 & 0,124 & 0,867 & 0,165 & 0,168 \\
\hline Coluna lombar & 1,063 & 0,180 & 1,136 & 0,232 & 0,222 \\
\hline
\end{tabular}

* Teste t de Student

Tabela 9

Média e D. P. da densidade mineral óssea em diferentes localizações segundo a relação LDL colesterol/HDL colesterol (Índice de Castelli 2).

\begin{tabular}{|c|c|c|c|c|c|}
\hline \multirow[t]{3}{*}{ Localização } & \multicolumn{4}{|c|}{ Castelli $2(n=70)$} & \multirow[t]{3}{*}{$p^{*}$} \\
\hline & \multicolumn{2}{|c|}{$<3,2(n=64)$} & \multicolumn{2}{|c|}{$\geq 3,2(n=6)$} & \\
\hline & $\mathrm{X}$ & D. P. & $x$ & D. P. & \\
\hline Colo de fêmur & 0,922 & 0,132 & 1,067 & 0,184 & 0,272 \\
\hline Triângulo de Wards & 0,788 & 0,145 & 0,929 & 0,248 & 0,062 \\
\hline Trocânter & 0,759 & 0,129 & 0,895 & 0,183 & 0,249 \\
\hline Coluna lombar & 1,057 & 0,178 & 1,306 & 1,528 & 0,749 \\
\hline
\end{tabular}

*Teste $\mathrm{t}$ de Student 


\section{Tabela 10}

Variáveis do perfil lipídico correlacionadas à densidade mineral óssea nas localizações (análise múltipla).

\begin{tabular}{|c|c|c|c|}
\hline Variáveis do perfil lipídico & Coeficiente & E.P. coeficiente & $\mathrm{p}$ \\
\hline \multicolumn{4}{|l|}{ Colo } \\
\hline Colesterol $\geq 240 \mathrm{mg} \%$ & $-0,126$ & 0,052 & 0,017 \\
\hline Índice de Castelli 2 & 0,058 & 0,027 & 0,034 \\
\hline Constante & 0,842 & 0,055 & $<0,001$ \\
\hline \multicolumn{4}{|l|}{ Triângulo } \\
\hline Colesterol $\geq 240 \mathrm{mg} \%$ & $-0,146$ & 0,057 & 0,013 \\
\hline Índice de Castelli 2 & 0,007 & 0,029 & 0,011 \\
\hline Constante & 0,665 & 0,061 & $<0,001$ \\
\hline \multicolumn{4}{|l|}{ Trocânter } \\
\hline Colesterol $\geq 240 \mathrm{mg} \%$ & $-0,113$ & 0,049 & 0,026 \\
\hline Índice de Castelli 2 & 0,079 & 0,026 & 0,002 \\
\hline Constante & 0,622 & 0,053 & $<0,001$ \\
\hline \multicolumn{4}{|l|}{ Coluna lombar } \\
\hline Colesterol $\geq 240 \mathrm{mg} \%$ & $-0,111$ & 0,087 & 0,205 \\
\hline Índice de Castelli 2 & 0,085 & 0,045 & 0,064 \\
\hline Constante & 0,927 & 0,093 & $<0,001$ \\
\hline
\end{tabular}

Figura 1

Curva ROC do colesterol total como preditor de diminuição da DMO no colo do fêmur e na coluna lombar.

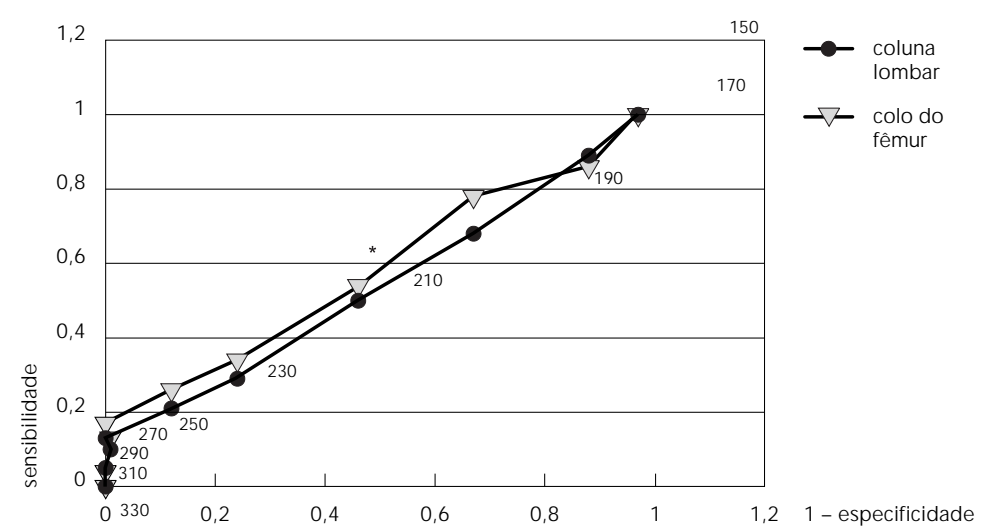

\begin{tabular}{lllllllllll} 
& A & B & C & D & E & F & G & H & I & J \\
\hline Valor de x & 0 & 0 & 0,009 & 0 & 0,12 & 0,24 & 0,46 & 0,67 & 0,88 & 0,97 \\
Y - Coluna lombar & 0 & 0,05 & 0,1 & 0,13 & 0,21 & 0,29 & 0,5 & 0,68 & 0,89 & 1 \\
Y - Colo do fêmur & 0 & 0,04 & 0,13 & 0,17 & 0,26 & 0,34 & 0,54 & 0,78 & 0,86 & 1
\end{tabular}

* Ponto com sensibilidade de $50 \%$ e especificidade de $54 \%$, o mais próximo do canto superior esquerdo da figura. máticos elevados de colesterol total associaram-se a menor DMO, ao passo que níveis elevados da relação lipoproteína de baixa e alta densidades, ou seja, também de risco para doenças cardiovasculares, associaram-se a maior densidade mineral óssea.

Cabe considerar também que, para qualquer valor das categorias do perfil lipídico avaliadas, encontraram-se sensibilidade e especificidade baixas em relação à DMO, para um mesmo valor. Em outras palavras, a capacidade dos níveis de colesterol total e de algumas de suas frações em predizer a ocorrência de menores valores de DMO foi muito baixa. Também foi baixa sua capacidade de diagnosticar que não existe diminuição da massa óssea. 0 ponto de corte utilizado para as variáveis do perfil lipídico no cálculo de sua sensibilidade e especificidade como indicativo da massa óssea foi obtido através da curva ROC, que representa a relação entre a sensibilidade e a especificidade dos diferentes valores das categorias do perfil lipídico. O valor ponto de corte com sensibilidade e especificidade mais bem equilibradas é o que se encontra mais próximo do ângulo superior esquerdo. Quanto mais retilínea for a curva, quanto mais próxima à diagonal, pior o desempenho do teste.

Pode-se considerar que um dos pontos importantes para a inconsistência dos resultados deste estudo deve-se, provavelmente, às diferenças na prevalência de alterações no perfil lipídico, em relação tanto aos estudos nacionais, como aos estudos internacionais. Em estudos anteriores do ambulatório de menopausa do CAISM-Unicamp observou-se que o colesterol total estava normal em 90,4\% dos 235 casos avaliados, com LDL colesterol, HDL colesterol e LDL colesterol sem alterações em $99,1 \%, 96,8 \%$ e $86,3 \%$ das mulheres, respectivamente (Pinto Neto et al., 1991), situação diferente da observada por outros autores que realizaram estudos populacionais em outras regiões, inclusive na região metropolitana de São Paulo, que detectaram cerca de $15 \%$ de colesterol total sérico acima dos valores da normalidade (Martins, 1989; Bagbozan et al., 1993; Soo et al., 1993). Um outro ponto que também merece ser sempre lembrado refere-se à variação amostral, o que pode ter ocorrido nesta pesquisa.

Em relação à osteoporose, relembramos que a casuística deste estudo foi representada principalmente por mulheres com idade abaixo de 55 anos e recém-menopausadas, com poucos fatores de risco para esta patologia; portanto, esperava-se elevado percentual de pacientes com densidade mineral óssea nor- 
mal nos distintos locais avaliados. Isto também pode explicar as diferenças nos resultados observados em relação aos estudos de Soo et al. (1993) e Bagbozan et al. (1993), bem como diferenças nas características da população estudada.

Faz-se importante, ainda, ressaltar um dado inesperado que ocorreu neste estudo. Observou-se associação inversa entre o HDL colesterol e a massa óssea quando se testou a diferença entre as médias da densidade mineral óssea nas diferentes localizações e os valores estratificados desta variável. Um perfil de risco para doenças cardiovasculares apresenta níveis mais baixos de HDL colesterol, concomitantemente com elevação dos níveis de LDL colesterol e de colesterol total. Esse foi um resultado inesperado, pois não houve associação com as outras frações do perfil lipídico quando se utilizou o mesmo cálculo estatístico, e mais inesperado ainda por ser uma associação inversa, ou seja, quanto melhor for o perfil lipídico (maiores níveis de HDL colesterol), menores os valores de DMO observados.

Na análise de regressão múltipla, observouse também uma associação direta entre o índice de Castelli 2 e a massa óssea. Como o índice de Castelli 2 representa a relação do LDL/ HDL colesterol, esperava-se encontrar valores elevados destes associados com menores valores de DMO, o que não ocorreu. Logo, este resultado também foi inconsistente. Não incluímos no modelo de regressão algumas variáveis possivelmente confundidoras, como, por exemplo, a idade, uma vez que a grande maioria das pacientes estava na faixa etária dos 46 a 55 anos, tendo-se, dessa forma, um grupo de pacientes não heterogêneo em relação a esta variável. A mesma consideração pode ser feita em relação ao tempo de pós-menopausa: o tempo médio de pós-menopausa foi de quatro anos, observando-se maior percentual de mulheres menopausadas há menos de seis anos.

Finalmente, entendemos que $o$ atendimento à mulher menopausada numa realidade como a brasileira, onde os núcleos de assistência médica em nível primário - responsáveis pelo atendimento da maior parte da população não estão suficientemente equipados para os diagnósticos e nem adequadamente orientados em relação às diferentes perspectivas terapêuticas, deve basear-se nos aspectos preventivos em relação a todas as doenças que acometem pessoas nessa faixa etária, priorizandose a prevalência dos fatores de risco identificados. Apesar do papel que o perfil lipídico ocupa para a ocorrência das doenças cardiovascu-

\section{Figura 2}

Curva ROC do HDL colesterol como preditor de menor DMO no colo do fêmur e na coluna lombar.

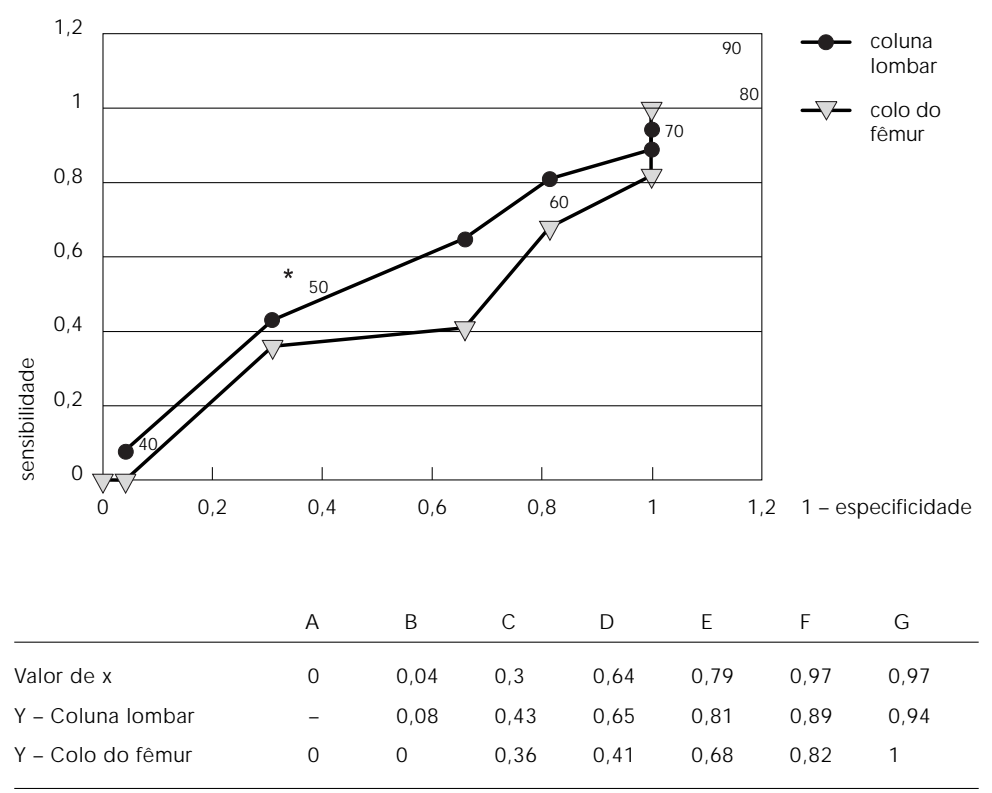

* Ponto com sensibilidade de $43 \%$ e especificidade de $70 \%$, o mais próximo do canto superior esquerdo da figura.

Figura 3

Curva ROC do LDL colesterol como preditor de diminuição de DMO no colo do fêmur e na coluna lombar.

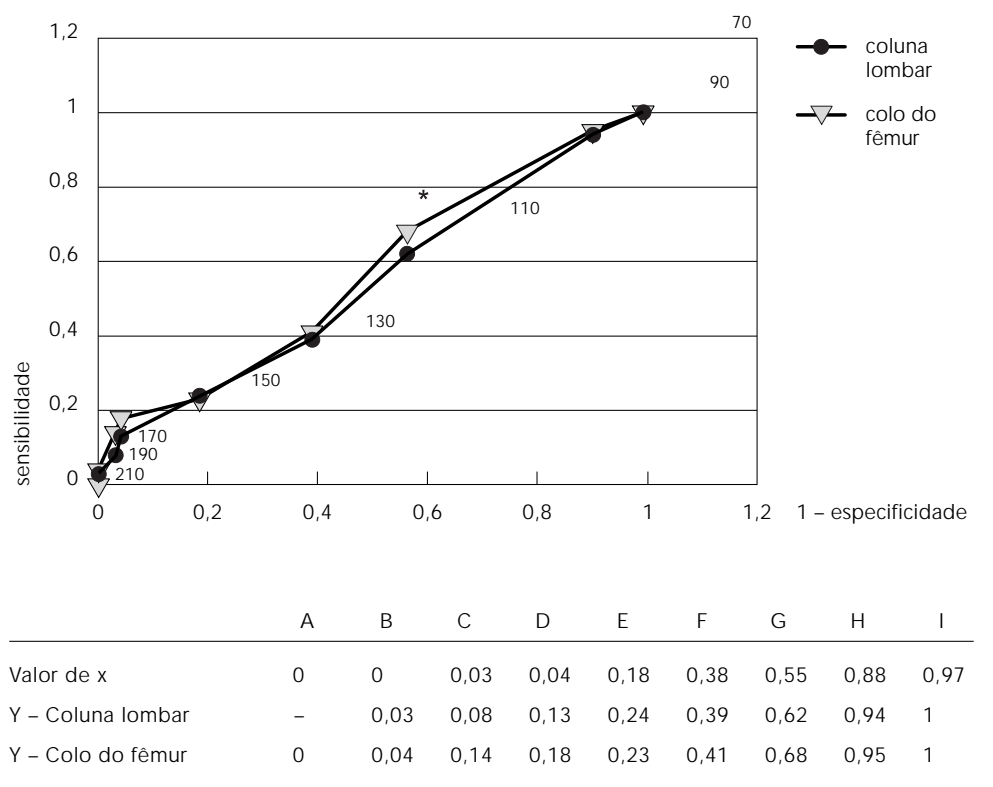

* Ponto com sensibilidade de $62 \%$ e especificidade de $45 \%$, o mais próximo do canto superior esquerdo da figura. 
lares, não recomendamos sua quantificação sistematicamente. Em relação à osteoporose, também consideramos que a densitometria óssea não deve ser realizada de rotina (Cummings \& Black, 1986). A prevenção de doenças cardiovasculares e osteoporose deve ser centrada rotineiramente no reconhecimento dos fatores de risco e mudança de hábitos de vida

\section{Referências}

BAGBOZAN, G.; BURSALI, A.; SERMET, A. \&TASKIN, V., 1993. The relation between serun lipid levels and postmenopause bone mass. Anais do VII Congresso Internacional de Menopausa de Estocolmo. Estocolmo: The International Menopause Society.

COX, D. R., 1970. The Analysis of Binary Data. London: Matheun \& CO. Ltd.

CUMMINGS, S. R. \& BLACK, M. A. D., 1986. Should perimenopausal women be screened for osteoporosis? Annals of Internal Medicine, 104:817823.

FERNANDES, C. E. \& PEREIRA FILHO, A. S., 1995. Climatério - Manual de Orientação. Rio de Janeiro: Federação Brasileira das Sociedades de Ginecologia e Obstetrícia.

FLETCHER, R. H.; FLETCHER, S. W. \&WAGNER, E. H., 1991. Epidemiologia Clínica: Bases Científicas da Conduta Médica. Porto Alegre: Artes Médicas.

KISH, L., 1965. Survey Sampling. New York: John Willey \& Sons.

LOBO, R. A., 1994. Treatment of the postmenopausal woman: where we are today. In: Treatment of the Postmenopausal Woman: Basic and Clinical Aspects (R. A. Lobo, ed.), pp. 427-432, New York: Raven Press Ltd.

MARQUES NETO, J. F. \& LEDERM AN, R., 1995. Osteoporose: Brasil Ano 2000. São Paulo: Limay.
(Paiva et al., 1995; Fernandes \& Pereira Filho, 1995; Lobo, 1994; Marques Neto \& Lederman, 1995). Também pensamos que, entre outros pontos, a não-confirmação de uma hipótese não significa que não se deva insistir no uso de recursos nesta linha de pesquisa. Acreditamos que, pela importância do assunto, novos estudos devam ser efetuados.

MARTINS, I. S., 1989. Lipídios e alguns fatores de risco associados em uma população periférica da região metropolitana de São Paulo, SP - Brasil. Um estudo-piloto. Revista de Saúde Pública, 23: 236-243.

PAIVA, L. C.; PINTO NETO, A. M.; PERROTI, M. A.; PEDRO, A. O. \& LANE, E., 1995. Influência dos fatores de risco para osteoporose sobre a massa óssea de mulheres climatéricas. I Congreso Latino Americano de Climaterio y Menopausia - Flascym'95 Anais. Buenos Ayres: Flascym (Federação Latinoamericana das Sociedades de Climatério e Menopausa).

PINTO NETO, A. M.; NASCIMENTO, F. L. B.; PAIVA, L. H. S. C.; MIRANDA, W. A.; ZABAGLIA, S. F. C. \& LANE, E., 1991. Perfil lipídico na menopausa. Jornal Brasileiro deGinecologia, 101:249-252.

SOO, H. C.; SAM, H. C.; YIOUN, Y. H. \& JOU, A. L. 1993. Relationship between spinal bone mineral density and serun lipid profile in postmenopausal women. Anais do VII Congresso Internacional de Menopausa de Estocolmo. Estocolmo: The Internatiional Menopause Society.

SOUZA, M. H. \& SCHNEIDER, S. A. V., 1993. Tamanho Amostral. Campinas: Cemicamp (Centro de Pesquisa das Doenças Materno-Infantis de Campinas). 